\title{
Effectiveness of initiating treatment with valsartan/hydrochlorothiazide in patients with stage-1 or stage-2 hypertension
}

\author{
DH Zappe ${ }^{1}$, BF Palmer ${ }^{2}$, DA Calhoun ${ }^{3}$, D Purkayastha ${ }^{1}$, R Samuel ${ }^{1}$ and KA Jamerson ${ }^{4}$ \\ ${ }^{1}$ Clinical Development \& Medical Affairs, Novartis Pharmaceuticals Corporation, East Hanover, NJ, USA; \\ ${ }^{2}$ Division of Nephrology, Department of Internal Medicine, University of Texas at Southwestern Medical \\ Center at Dallas, Dallas, TX, USA; ${ }^{3}$ Vascular Biology and Hypertension Program, Division of Cardiovascular \\ Disease, University of Alabama at Birmingham, Birmingham, AL, USA and ${ }^{4}$ Division of Hypertension, \\ Department of Internal Medicine, University of Michigan, Ann Arbor, MI, USA
}

This prospective, 6-week, multicenter, double-blind study examined the benefits of initiating treatment with combination valsartan/hydrochlorothiazide (HCTZ) compared with initial valsartan monotherapy for 648 patients with stage-1 or stage-2 hypertension (age $=\mathbf{5 2 . 6} \pm 10$ years; $54 \%$ male; baseline blood pressure $(B P)=161 /$ $98 \mathrm{~mm} \mathrm{Hg}, 32 \%$ stage 1). Patients were randomized to valsartan $80 \mathrm{mg}$ (V-low), valsartan $160 \mathrm{mg}$ (V-high) or valsartan/HCTZ $160 / 12.5 \mathrm{mg}$ (V/HCTZ), and electively titrated after weeks 2 and 4 to the next dosage level (maximum dose valsartan/HCTZ 160/25 mg) if BP remained $>140 / 90 \mathrm{~mm} \mathrm{Hg}$. At end of the study, patients initiated with V/HCTZ required less titration steps compared with the initial valsartan monotherapy groups (63 vs $86 \%$ required titration by study end, respectively) and reached the target BP goal of $<140 / 90 \mathrm{~mm} \mathrm{Hg}$ in a shorter period of time $(2.8$ weeks) $(P<0.0001)$ vs V-low $(4.3$ weeks) and
V-high (3.9 weeks). Initial combination therapy was also associated with higher BP control rates and greater reductions in both systolic and diastolic BP from baseline $(63 \%,-27.7 \pm 13 /-15.1 \pm 8 \mathrm{~mm} \mathrm{Hg})$ compared with V-low (46\%, $-21.2 \pm 13 /-11.4 \pm 8 \mathrm{~mm} \mathrm{Hg}, P<0.0001)$ or $V-h i g h$ (51\%, $-24.0 \pm 13 /-12.0 \pm 10 \mathrm{~mm} \mathrm{Hg}, P<0.01)$. Overall and drug-related AEs were mild to moderate and were similar between V/HCTZ (53.1 and 14.1\%, respectively) and the two monotherapy groups, V-low (50.5 and 13.8\%) and V-high (50.7 and $11.8 \%)$. In conclusion, initiating therapy with a combination of valsartan and low-dose HCTZ results in early, improved BP efficacy with similar tolerability as compared with starting treatment with a low or higher dose of valsartan for patients with stage-1 and stage-2 hypertension.

Journal of Human Hypertension (2010) 24, 483-491; doi:10.1038/jhh.2009.90; published online 10 December 2009

Keywords: valsartan; valsartan/hydrochlorothiazide; initial combination therapy

\section{Introduction}

Based on recent national surveys, blood pressure (BP) control rates for patients with hypertension continue to be low and improvements in therapeutic management strategies are required in order for more patients to reach target BP goals. ${ }^{1}$ It is well recognized that most patients with hypertension will require two or more antihypertensive agents for effective BP control. ${ }^{2}$ Rational combinations of antihypertensive agents as single-pill formulations are readily available, but their use remains low primarily due to health care providers who are reluctant to intensify therapy. ${ }^{3}$ Earlier and more

Correspondence: Dr DH Zappe, Clinical Development \& Medical Affairs, Novartis Pharmaceuticals Corporation, One Health Plaza, East Hanover, NJ 07936, USA.

E-mail: dion.zappe@novartis.com

Received 27 June 2009; revised 6 October 2009; accepted 1 November 2009; published online 10 December 2009 frequent use of combination therapy in the management of hypertension would greatly improve BP control rates. The FDA recently approved single-pill combinations of an angiotensin-receptor blocker (ARB) and hydrochlorothiazide (HCTZ), ${ }^{4,5}$ and combinations of ARB with amlodipine for initial treatment of hypertension in patients whose BP would not be controlled on monotherapy alone. The initial therapy indication did not stipulate the BP level at which combination therapy should be initiated, but current treatment guidelines suggest that use of combination therapy should be guided by baseline $\mathrm{BP}$ and the cardiovascular (CV) risk status of the patient.,

For patients unresponsive to valsartan or lowdose HCTZ monotherapy, addition of the other agent has resulted in further reductions of $\mathrm{BP}$, without increase in adverse events (AEs). ${ }^{8-11}$ The excellent tolerability and complementary BP-lowering effects of this combination makes it an ideal agent for initial use in patients with stage-1 and stage-2 hypertension. The primary arguments against initiating 
treatment with combination therapy for hypertension are based on clinical need and safety concerns. Initial combination therapy for patients whose BP could be controlled by monotherapy, may lead to excessive drops in $\mathrm{BP}$ and thus increase the risk for hypotension-related AEs. Common practice is to initiate treatment with low-dose monotherapy and up-titrate to higher dose every 4 weeks. There has been no prospective study that has evaluated initial combination therapy using valsartan and HCTZ as compared with monotherapy when evaluating BP control rates and time to BP control in patients with primary essential hypertension without additional $\mathrm{CV}$ risk factors. Based on the need for improved $\mathrm{BP}$ control strategies, we conducted a study to determine the efficacy of initial combination therapy with valsartan and HCTZ for patients with stage-1 or stage-2, uncomplicated hypertension as compared with initiating therapy with low $(80 \mathrm{mg})$ or higher dose (160 mg) valsartan monotherapy.

\section{Patients and methods}

\section{Patients}

The study enrolled patients $\geqslant 18$ years of age with a documented diagnosis of hypertension defined as mean sitting systolic BP (MSSBP) $\geqslant 150$ and $<180 \mathrm{mmHg}$ and mean sitting diastolic BP (MSDBP) $\geqslant 90$ and $<110 \mathrm{~mm} \mathrm{Hg}$. Patients were excluded if they had severe hypertension, known or suspected secondary hypertension, previous myocardial infarction, stroke or other CV complications, severe hepatic disease, history of malignancy, or allergy/hypersensitivity to valsartan or HCTZ. Women of childbearing potential were eligible if pregnancy testing was negative. The protocol was approved by all relevant ethics committees and all patients provided written informed consent.

\section{Study design}

This was a 6-week, multicenter, randomized, double-blind, parallel-group study conducted across 78 US centres. Following a 14- to 28-day washout period, during which all pre-study antihypertensive medications, if used, were discontinued; eligible patients entered a 6-week, double-blind period during which they received valsartan (80 mg), valsartan $(160 \mathrm{mg})$ or valsartan $(160 \mathrm{mg}) / \mathrm{HCTZ}$ $(12.5 \mathrm{mg}$ ) for 2 weeks according to the randomization scheme (Figure 1). The three groups were referred to as valsartan-low (V-low), valsartan-high (V-high) and the combination group, valsar$\tan +$ HCTZ (V/HCTZ), respectively. At the end of this period, patients whose BP was uncontrolled (MSSBP $\geqslant 140 \mathrm{~mm} \mathrm{Hg}$ or $\mathrm{MSDBP} \geqslant 90 \mathrm{~mm} \mathrm{Hg}$ ) were titrated to the next dosage level (valsartan $160 \mathrm{mg}$, valsartan + HCTZ 160/12.5 mg, or valsartan + HCTZ 160/25 mg respectively in the 3 groups) for 2 weeks. Patients whose BP was uncontrolled
$(\mathrm{MSSBP} \geqslant 140 \mathrm{~mm} \mathrm{Hg}$ or $\mathrm{MSDBP} \geqslant 90 \mathrm{~mm} \mathrm{Hg}$ ) at week 4, were titrated to the next dosage level (valsartan + HCTZ 160/12.5 mg or valsartan + HCTZ $160 / 25 \mathrm{mg}$ ) in the V-low and V-high groups while V/HCTZ was maintained at the dose of $160 / 25 \mathrm{mg}$ (Figure 1). Patients achieving a target BP goal of $<140 / 90 \mathrm{~mm} \mathrm{Hg}$ were maintained at the dose level to which they responded during the double-blind treatment phase. Patients were instructed to take their medication in the morning at the same time each day. To maintain the blinded nature of the study, patients on monotherapy received one placebo pill along with a pill of active therapy.

Concomitant therapy with the following medications was prohibited: other ARBs, angiotensinconverting enzyme (ACE) inhibitors, $\beta$-adrenergic antagonists, calcium-channel blockers, any other antihypertensive(s), and potassium-sparing diuretics (for example, spironolactone, triamterene or amiloride), anti-arrhythmic drugs, nitrates, $\alpha$-adrenergic antagonists and digitalis glycosides. Drugs to treat erectile dysfunction were permitted, except within $24 \mathrm{~h}$ (sildenafil, vardenafil) and $48 \mathrm{~h}$ (tadalafil) of study visits.

\section{Outcomes}

The objectives of this trial were to compare the proportion of patients achieving BP control and the change in MSSBP and MSDBP from baseline between antihypertensive treatment regimens, initiated with valsartan ( $80 \mathrm{mg})$, valsartan $(160 \mathrm{mg}$ ) or valsartan + HCTZ (160/12.5 mg). The primary efficacy variable was change in MSSBP from baseline to week 4 . Secondary outcomes included time to achieve BP goal (defined as the first achievement of the target BP goal of $<140 \mathrm{~mm} \mathrm{Hg}$ systolic BP and $<90 \mathrm{~mm} \mathrm{Hg}$ diastolic BP during the 6-week period), in all patients and in patients with stage-1 (SBP $140-159 \mathrm{~mm} \mathrm{Hg}$ or DBP $90-99 \mathrm{~mm} \mathrm{Hg}$ ) and stage-2 hypertension (SBP $\geqslant 160 \mathrm{~mm} \mathrm{Hg}$ or $\mathrm{DBP} \geqslant 100 \mathrm{~mm} \mathrm{Hg}$ ).

During study visits, BP and heart rate measurements were obtained and AEs that occurred since the last study visit were recorded. Any unfavourable medical occurrence, regardless of its suspected cause, was considered an AE. The frequency and severity of AEs, as well as their relationship to study medication, were summarized.

\section{Statistical methods}

The sample size was based on the comparison of treatment effects between patients initiated with valsartan $(80 \mathrm{mg})$ and those initiated with valsartan $(160 \mathrm{mg}$ ) with respect to the primary efficacy variable, change in MSSBP from baseline to week 4 . Assuming a between-group difference in change in mean MSSBP from baseline to week 4 of the doubleblind treatment period of 4.0 (s.d. $=14 \mathrm{~mm} \mathrm{Hg}$ ), it was projected that 216 patients per treatment group (a total sample size of 648 randomized patients) would provide an $80 \%$ power to detect a 

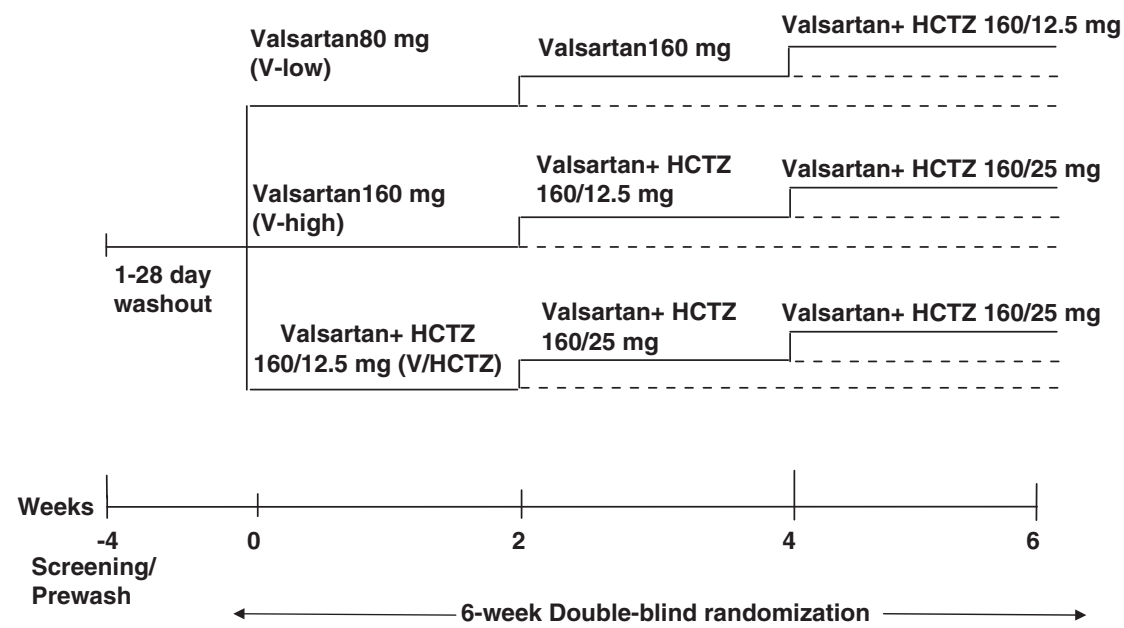

Figure 1 Study design.

statistically significant difference at the 0.05 level (two-sided). To control for overall type-I error, a stepwise testing procedure for the primary analyses was adopted. The primary efficacy comparison between the V-low group and the V-high group was tested first. Primary efficacy comparison between V-low and V/HCTZ was performed only if test result from V-low group vs V-high group was statistically significant.

The safety population consisted of all randomized subjects who received at least one dose of the study medication. All efficacy analyses were performed using the intent-to-treat population (all randomized patients who received at least one dose of the study drug and had a valid baseline and at least one valid post-baseline assessment of the primary efficacy variable). For analysis of efficacy data, a lastobservation carried forward (LOCF) method was used. An analysis of covariance model was used with baseline measurement as a covariate and treatment as a factor in the model for analyses of all continuous primary and secondary efficacy variables. The primary time point for the analyses was week 4. $\chi^{2}$-Tests were used for categorical variables (the number and percentage of patients achieving BP goal). The time to achieving BP control was analysed by Kaplan-Meier estimates and was presented for each treatment arm. Wilcoxon tests were used to test the differences between treatments.

\section{Results}

Patient disposition

From a total of 1413 patients screened for the study, 652 patients were enrolled in the double-blind phase and randomly assigned to one of the three treatment groups: V-low $(n=218)$, V-high $(n=221)$ and V/HCTZ $(n=213)$. Most patients who participated in the double-blind treatment phase were Caucasian $(67 \%)$, with a mean age of 53 years and almost $88 \%$ of the patients $<65$ years of age. Most of the patients were not diabetic $(87 \%)$, but a majority had the metabolic syndrome $(57 \%)$. At least half of all patients enrolled were on previous antihypertensive therapy. Overall, demographic characteristics were similar between the treatment groups (Table 1).

Of the 652 patients randomized into the study, 51 withdrew prior to completing their participation: V-low, $n=17$; V-high, $n=17$ and V/HCTZ, $n=17$. The most common reasons for patient withdrawal were AEs, unsatisfactory therapeutic effect and patient withdrawal of consent. A total of four patients (1 patient in the V-high group and 3 patients in the V/HCTZ group) were excluded from the intentto-treat population due to missing baseline or post-baseline assessments. Patient disposition throughout the study is illustrated in Figure 2. Most of the patients followed the treatment regimen as prescribed, with the exception of 37 patients (10 patients in the V-low group, 15 patients in the V-high group and 12 patients in the V/HCTZ group) with reported missed doses, titration errors or intake of prohibited medications. During the study $87 \%$ of patients in the V-low group and $86 \%$ of patients in the V-high group required titration to the next dose level (level 2 or level 3 ) as compared with $63 \%$ of patients for V/HCTZ.

\section{Efficacy outcomes}

The proportion of patients achieving a BP control rate $<140 / 90 \mathrm{~mm} \mathrm{Hg}$ was higher $(P<0.05)$ for the V/ HCTZ group as compared with the V-low and V-high group at weeks 2, 4 and 6 (Figure 3). Significant differences between the V-high group and V-low group regarding the percentage of patients achieving the target BP goal of $<140 / 90 \mathrm{~mm} \mathrm{Hg}$ at week 4 (46 vs $26 \%$, respectively; $P<0.0001$ ), and numerically higher number of patients achieving the BP goal in the V-high group at week 6 ( 51 vs $45 \%$, respectively; $P=$ NS) was observed. This was primarily attributed to the addition of HCTZ. Within each treatment group, 
Table 1 Patient characteristics at baseline during the doubleblind treatment period

\begin{tabular}{|c|c|c|c|}
\hline & \multicolumn{3}{|c|}{ Treatment groups } \\
\hline & V-low & V-high & V/HCTZ \\
\hline$n$ & 218 & 221 & 213 \\
\hline $\begin{array}{l}\text { Age (years) } \\
\quad \geqslant 65 \text { years, } n(\%)\end{array}$ & $\begin{array}{l}52.2 \pm 10.7 \\
21(9.6)\end{array}$ & $\begin{array}{l}53.1 \pm 9 \\
25(11.3)\end{array}$ & $\begin{array}{l}52.6 \pm 10 . \\
30(14.1)\end{array}$ \\
\hline Female, $n(\%)$ & $99(45.4)$ & $98(44.3)$ & $101(47.4)$ \\
\hline \multicolumn{4}{|l|}{ Race, n (\%) } \\
\hline Caucasian & $152(69.7)$ & $150(67.9)$ & $137(64.3)$ \\
\hline Black & $37(17.0)$ & $38(17.2)$ & $39(18.3)$ \\
\hline Hispanic & $21(9.6)$ & $22(10.0)$ & $23(10.8)$ \\
\hline $\begin{array}{l}\text { Previous antihypertensive } \\
\text { medication (last } 30 \text { days), } \\
n(\%)\end{array}$ & $108(49.5)$ & $128(57.9)$ & $106(49.8)$ \\
\hline BW (kg) & $92.7 \pm 21$ & $94.2 \pm 21$ & $93.2 \pm 20$ \\
\hline BMI $\left(\mathrm{kg} \mathrm{m}^{-2}\right)$ & $32.0 \pm 6.8$ & $32.7 \pm 6.4$ & $32.5 \pm 6.9$ \\
\hline Potassium $\left(\mathrm{mmol} \mathrm{l}^{-1}\right)$ & $4.36 \pm 0.5$ & $4.27 \pm 0.4$ & $4.29 \pm 0.4$ \\
\hline Serum creatinine $\left(\mathrm{mg} \mathrm{dl}^{-1}\right)$ & $0.87 \pm 0.2$ & $0.89 \pm 0.2$ & $0.86 \pm 0.2$ \\
\hline Glucose $\left(\mathrm{mg} \mathrm{dl}^{-1}\right)$ & $107 \pm 38$ & $107 \pm 38$ & $107 \pm 34$ \\
\hline Total cholesterol $\left(\mathrm{mg} \mathrm{dl}^{-1}\right)$ & $205 \pm 41$ & $200 \pm 44$ & $204 \pm 39$ \\
\hline LDL-C (mg dl $\left.{ }^{-1}\right)$ & $121.5 \pm 33$ & $119 \pm 35$ & $122 \pm 32$ \\
\hline HDL-C (mg dl-1) & $51.5 \pm 14$ & $50.4 \pm 13$ & $52.6 \pm 14$ \\
\hline Triglycerides (mg dl $\left.{ }^{-1}\right)$ & $161 \pm 99$ & $157 \pm 127$ & $154 \pm 93$ \\
\hline Metabolic syndrome, $n(\%)$ & $125(57.3)$ & $126(57.0)$ & $121(56.8)$ \\
\hline Diabetes* $^{*} n(\%)$ & $23(10.6)$ & $30(13.6)$ & $30(14.1)$ \\
\hline \multicolumn{4}{|l|}{ Mean sitting $B P(\mathrm{~mm} \mathrm{Hg})$} \\
\hline Systolic & $160.7 \pm 7.4$ & $161.1 \pm 7.9$ & $161.0 \pm 7.8$ \\
\hline Diastolic & $97.7 \pm 5.5$ & $98.2 \pm 5.1$ & $98.2 \pm 5.1$ \\
\hline $\begin{array}{l}\text { Stage-2 hypertension, } \\
n(\%)\end{array}$ & $147(67.4)$ & $148(67.3)$ & $145(69.0)$ \\
\hline
\end{tabular}

Abbreviations: BMI, body mass index; BP, blood pressure; BW, body weight; HDL-C, high-density lipoprotein cholesterol; LDL-C, lowdensity lipoprotein cholesterol; V/HCTZ, valsartan/hydrochlorothiazide; V-high, valsartan $160 \mathrm{mg}$; V-low, valsartan $80 \mathrm{mg}$.

*Diabetes defined as fasting plasma glucose $\geqslant 126 \mathrm{mg} \mathrm{dl}^{-1}$ $\left(7.0 \mathrm{mmol} \mathrm{l}^{-1}\right)$. Data represented as mean \pm s.d. unless otherwise noted. the changes in MSSBP and MSDBP from baseline were statistically significant at weeks 2,4 and 6 (Figures 4a and b). At weeks 2, 4 and 6, the reduction in the MSSBP and MSDBP were greater $(P<0.01)$ in the V/HCTZ group as compared with reductions observed in both V-low and V-high groups. Change in MSSBP from baseline at week 4 (primary efficacy) was: $-15.3 \pm 12 \mathrm{~mm} \mathrm{Hg}$ for the V-low group; $-22.0 \pm 13 \mathrm{~mm} \mathrm{Hg}$ for the V-high group and $-26.0 \pm 14 \mathrm{~mm} \mathrm{Hg}$ for V/HCTZ. At week 6 maximal reduction in SBP from baseline was observed in the V/HCTZ group $(-27.7 \pm 13 \mathrm{~mm} \mathrm{Hg})$ and was greater $(P<0.01)$ when compared with that in the V-low $(-21.2 \pm 13 \mathrm{~mm} \mathrm{Hg}$, difference $=$

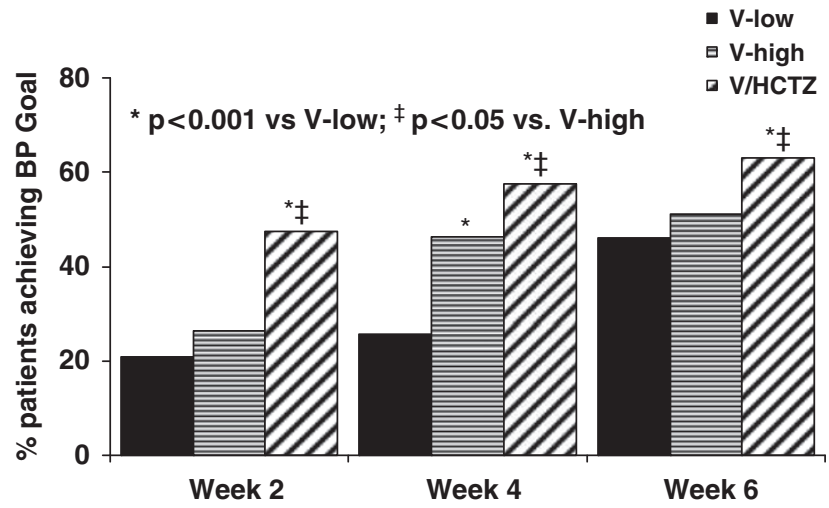

Figure 3 Percentage of patients achieving the BP goal $(<140 /$ $90 \mathrm{~mm} \mathrm{Hg}$ ). The primary efficacy time point was at week 4 . At all weeks, 2,4 and 6 , the difference in the percentage of patients achieving BP control between the V/HCTZ and V-low $(P<0.001)$ and between the V/HCTZ and V-high $(P<0.05)$ groups was statistically significant. The difference between V-low and V-high was significant $(P<0.001)$ only at week 4 but not at weeks 2 and 6 . BP, blood pressure; V/HCTZ, valsartan/hydrochlorothiazide; V-high, valsartan $160 \mathrm{mg}$; V-low, valsartan $80 \mathrm{mg}$.

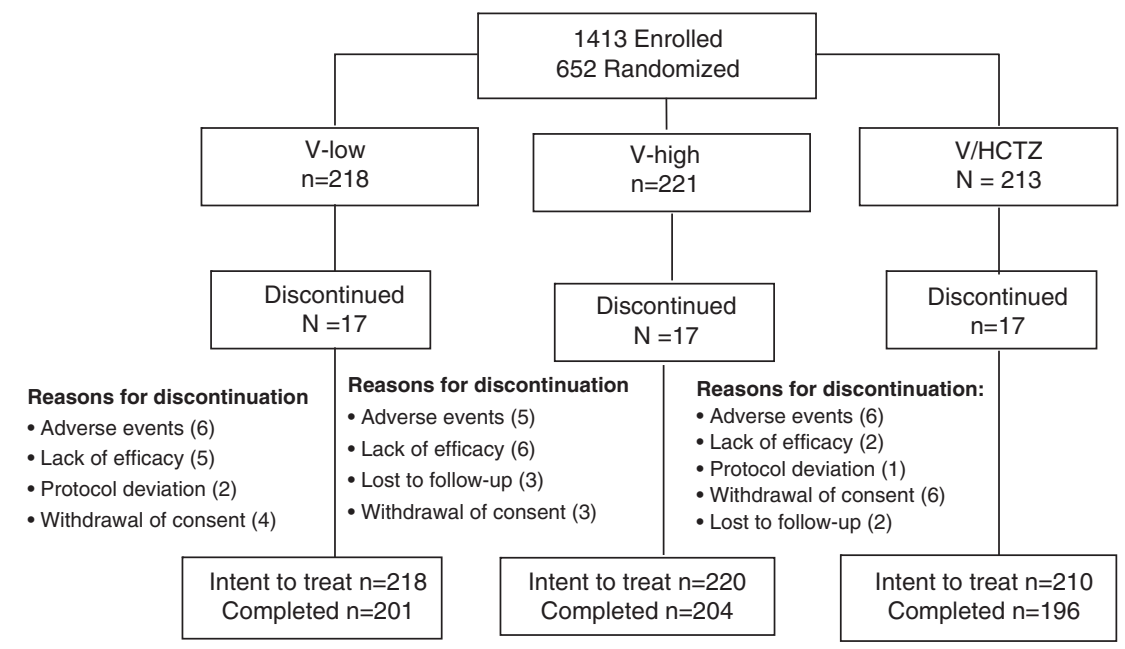

Figure 2 Patient disposition. V-low refers to patients initiated therapy with valsartan (80 mg); V-high refers to patients initiated therapy with valsartan (160 mg) and V/HCTZ refers to patients initiated with combination therapy. V/HCTZ, valsartan/hydrochlorothiazide. 


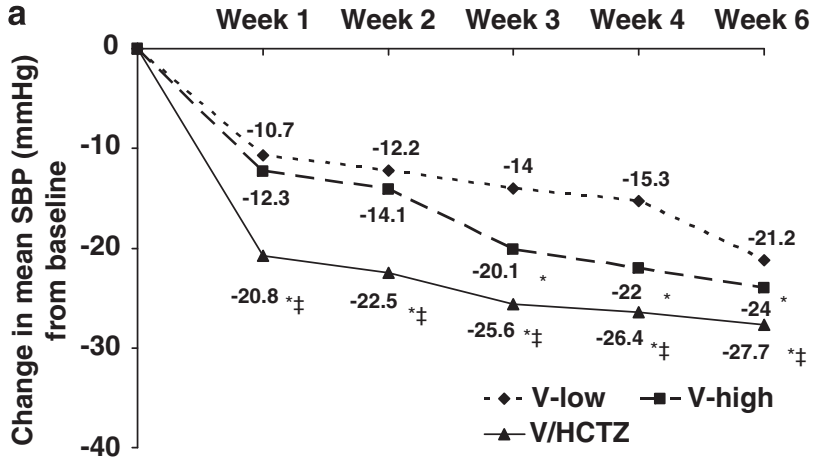

${ }^{*} p<0.001$ vs V-low; ${ }^{\ddagger} p<0.01$ vs. V-high

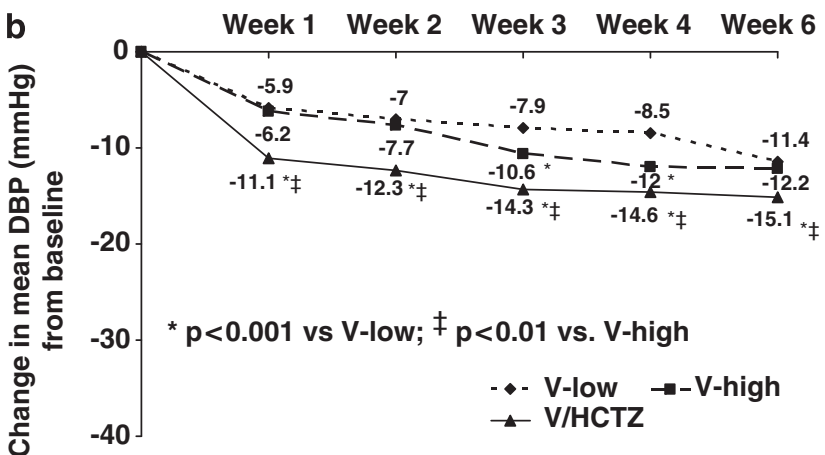

Figure 4 Change in (a) MSSBP ( $\mathrm{mm} \mathrm{Hg}$ ) and (b) MSDBP (mm Hg) from baseline to week 6 of the double-blind treatment period. The primary efficacy time point was at week 4 . At weeks 1, 2, 3, 4 and 6 , the reduction in MSSBP and MSDBP was significantly greater in the V/HCTZ group as compared with the reductions observed in both V-low $(P<0.001)$ and V-high groups $(P<0.01)$. Reductions in MSSBP were significantly greater with V-high as compared with V-low only at weeks 3,4 and $6(P<0.001)$ and reductions in MSDBP were significantly greater with V-high as compared with V-low only at weeks 3 and $4(P<0.001)$. BP, blood pressure; MSSBP, mean sitting systolic BP; MSSDP, mean sitting diastolic BP; V/HCTZ, valsartan/hydrochlorothiazide; V-high, valsartan $160 \mathrm{mg}$; V-low, valsartan $80 \mathrm{mg}$.

$6.6 \mathrm{~mm} \mathrm{Hg})$ and V-high $(-24.0 \pm 13 \mathrm{~mm} \mathrm{Hg}$, difference $=3.8 \mathrm{~mm} \mathrm{Hg}$ ) groups. The reductions in MSSBP observed in the V-high group were greater $(P<0.05)$ when compared with those observed with V-low group during both weeks 4 and 6 (Figure 4a).

The mean reductions in MSDBP from baseline to week 6 was greater $(P<0.001)$ in the V/HCTZ group $(-14.6 \pm 7 \mathrm{~mm} \mathrm{Hg})$ as compared with that in the V-low $(-8.5 \pm 7 \mathrm{~mm} \mathrm{Hg})$ and V-high groups $(-12.0 \pm 10 \mathrm{~mm} \mathrm{Hg})$ (Figure 4b). The reduction from baseline in MSDBP observed with V-high was greater $(P<0.001)$ at week $4(-7.7 \pm 9 \mathrm{~mm} \mathrm{Hg})$ as compared with V-low.

$\mathrm{BP}$ control rates and changes in MSSBP and MSDBP from baseline to week 6 in subgroups of patients with stage-1 or stage-2 hypertension were examined through post hoc analysis. Average baseline BP in the stage-1 hypertension group was 154/ $95 \mathrm{~mm} \mathrm{Hg}$ as compared with $164 / 100 \mathrm{~mm} \mathrm{Hg}$ in the stage-2 hypertension group. BP control rates after 4 weeks of therapy for patients with stage- 1 and stage2 hypertension were higher $(P<0.05)$ for V/HCTZ

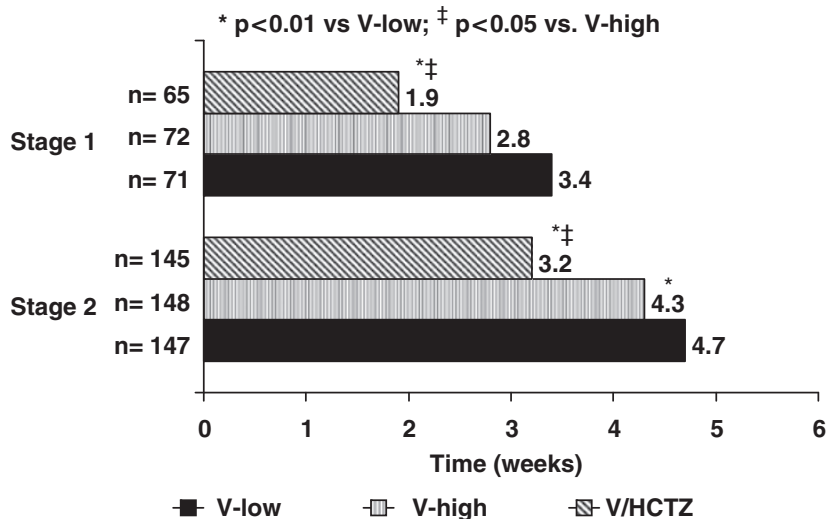

Figure 5 Mean time to BP goal defined as time (weeks) to achieving first treatment success $(\mathrm{BP}<140 / 90 \mathrm{~mm} \mathrm{Hg})$ in patients with stage- 1 and stage- 2 hypertension. $P<0.05$ for the V/HCTZ group as compared with the V-low and V-high group at week 6. BP, blood pressure; V/HCTZ, valsartan/hydrochlorothiazide; V-high, valsartan $160 \mathrm{mg}$; V-low, valsartan $80 \mathrm{mg}$.

(70 and $52 \%$, respectively) as compared with that for V-low (38 and 20\%, respectively). Reductions in SBP and DBP were greater in stage- 2 as compared with stage-1 hypertension among the treatment groups; however, the BP lowering advantage with V/HCTZ remained $(P<0.05)$ when compared with V-low and V-high for patients with either stage-1 or stage-2 hypertension. The systolic and diastolic BP difference between V/HCTZ and V-low at week 4 was $-9.2 /-6.1 \mathrm{~mm} \mathrm{Hg}$ in stage-1 and $-11.7 /-5.9 \mathrm{~mm} \mathrm{Hg}$ in stage-2.

Time to BP goal

The mean time to achieve $\mathrm{BP}$ goal, defined as first achievement of the target BP goal of $<140 /$ $90 \mathrm{~mm} \mathrm{Hg}$, was significantly improved $(P<0.0001)$ with V/HCTZ ( $2.8 \pm 0.13$ weeks) as compared with that in the V-high $(3.9 \pm 0.15$ weeks $)$ and V-low groups ( $4.3 \pm 0.14$ weeks) in both stage- 1 and stage- 2 hypertension (Figure 5). The median time to first treatment success (that is, the time point at which $50 \%$ of patients reached their goal) was 2.0 weeks (95\% confidence interval 2.0, 3.0) in the V/HCTZ group, compared with 6.0 weeks $(95 \%$ confidence interval $4.0,6.0$ ) in the V-low and 3.0 weeks (95\% confidence interval 3.0, 4.0) in the V-high group.

\section{Adverse events}

The three treatment regimens, valsartan $(80 \mathrm{mg})$, valsartan (160 mg) and valsartan/HCTZ (160/12.5 mg), were well-tolerated. There were no clinically or statistically significant differences in the AE rates among the three groups. Overall, AEs were experienced by $50.5 \%(110 / 218)$ of patients in the V-low group, $50.7 \%(112 / 221)$ of patients in the V-high group and $53.1 \%(113 / 213)$ of patients in the V/HCTZ group. Drug-related AEs were similar across the three treatment groups for V-low 
Table 2 Most frequent AEs

\begin{tabular}{|c|c|c|c|}
\hline & $\begin{array}{c}\text { V-low } \\
(\mathrm{n}=218)\end{array}$ & $\begin{array}{c}\text { V-high } \\
(\mathrm{n}=221)\end{array}$ & $\begin{array}{l}V / H C T Z \\
(\mathrm{n}=213)\end{array}$ \\
\hline Patients with AEs, $n(\%)$ & $110(50.5)$ & $112(50.7)$ & $113(53.1)$ \\
\hline $\begin{array}{l}\text { Suspected AEs related to } \\
\text { study drug, } n(\%)\end{array}$ & $30(13.8)$ & $26(11.8)$ & $30(14.1)$ \\
\hline $\begin{array}{l}\text { AEs leading to } \\
\text { discontinuation, } n(\%)\end{array}$ & $6(2.8)$ & $5(2.3)$ & $6(2.8)$ \\
\hline \multicolumn{4}{|l|}{$A E$ preferred term, $n(\%)$} \\
\hline Headache & $18(8.3)$ & $17(7.7)$ & $10(4.7)$ \\
\hline Dizziness & $11(5.0)$ & $20(9.0)$ & $17(8.0)$ \\
\hline Fatigue & $10(4.6)$ & $7(3.2)$ & 8 (3.8) \\
\hline Nausea & $8(3.7)$ & $7(3.2)$ & $9(4.2)$ \\
\hline Diarrhoea & $8(3.7)$ & $5(2.3)$ & $8(3.8)$ \\
\hline $\begin{array}{l}\text { Upper respiratory tract } \\
\text { infection }\end{array}$ & $5(2.3)$ & $7(3.2)$ & $7(3.3)$ \\
\hline Back pain & $0(0.0)$ & $3(1.4)$ & $7(3.3)$ \\
\hline Myalgia & $7(3.2)$ & $2(0.9)$ & $1(0.5)$ \\
\hline
\end{tabular}

Abbreviation: AE, adverse event.

aThe term dizziness includes any term associated with low BP, such as postural dizziness, hypotension, orthostatic hypotension and vertigo.

(13.8\%), V-high (11.8\%) and V/HCTZ (14.1\%). The most frequent $\mathrm{AEs}$, experienced by at least $3 \%$ of patients in any treatment group, were headache, dizziness, fatigue, nausea, diarrhoea, upper respiratory tract infection, back pain and myalgia (Table 2). Most AEs were considered by the investigator to be of mild or moderate severity and were not suspected to be treatment-related. AEs associated with low BP, such as dizziness (including postural dizziness), vertigo and hypotension (including orthostatic hypotension), were observed in $5 \%$ of patients with V-low, $9 \%$ of patients with V-high and $8 \%$ of patients with V/HCTZ. The incidence of AEs associated with low BP appeared to be higher for V/HCTZ and V-high as compared with V-low, but only three patients in V/HCTZ and V-high reported symptoms of moderate severity, compared with four in the V-low group; all else were mild.

Analysis of the most frequent AEs experienced by at least $5 \%$ of patients (headache and dizziness) showed an overall increased incidence in the first two weeks of the study compared with 4 and 6 weeks after therapy was initiated. At week 2 , the incidence of dizziness (overall 2.9\%; V-low 2.3\%; V-high 2.7\%; V/HCTZ $3.8 \%$ ) and headache (overall $4.6 \%$; V-low $5.5 \%$; V-high 5.9\%; V/HCTZ $2.3 \%$ ) were higher compared with those observed at week 4 (dizziness: overall $2.3 \%$; V-low $0.9 \%$; V-high $3.1 \%$; V/HCTZ $2.8 \%$ and headache: overall $1.5 \%$; V-low $2.3 \%$; V-high 1.4\%; V/HCTZ 0.9\%) and week 6 (dizziness: overall $0.9 \%$; V-low $1.4 \%$; V-high $1.8 \%$; V/HCTZ $0.5 \%$ and headache: overall $0.5 \%$; V-low $0.5 \%$; V-high $0.5 \%$; V/HCTZ 0.5\%).

There were no significant changes in any of the laboratory measures from baseline, including serum creatinine, glucose or lipid levels, during the study, except potassium levels (Table 3). Potassium levels decreased in all the groups, with significantly greater
Table 3 Blood chemistry measures after 6 weeks of treatment: change from baseline

\begin{tabular}{|c|c|c|c|}
\hline & \multicolumn{3}{|c|}{ Treatment groups } \\
\hline & V-low & V-high & V/HCTZ \\
\hline Potassium $\left(\mathrm{mmol} \mathrm{l}^{-1}\right)$ & $-0.09 \pm 0.5^{*}$ & $-0.11 \pm 0.4^{*}$ & $-0.19 \pm 0.4^{* \dagger}$ \\
\hline Serum creatinine $\left(\mathrm{mg} \mathrm{dl}^{-1}\right)$ & $0.02 \pm 0.1$ & $0.04 \pm 0.2$ & $0.04 \pm 0.1$ \\
\hline Glucose $\left(\mathrm{mg} \mathrm{dl}^{-1}\right)$ & $4.2 \pm 23$ & $2.3 \pm 30$ & $1.0 \pm 24$ \\
\hline Total cholesterol $\left(\mathrm{mg} \mathrm{dl}^{-1}\right)$ & $-4.5 \pm 28$ & $-1.4 \pm 29$ & $0.3 \pm 29$ \\
\hline LDL-C $\left(\mathrm{mg} \mathrm{dl}^{-1}\right)$ & $-5.9 \pm 23$ & $-3.9 \pm 27$ & $-1.9 \pm 23$ \\
\hline HDL-C (mg dl-1 $)$ & $-0.6 \pm 6$ & $-0.6 \pm 6$ & $-1.3 \pm 8$ \\
\hline Triglycerides (mg dl ${ }^{-1}$ ) & $9.6 \pm 78$ & $13.7 \pm 72$ & $20.1 \pm 98$ \\
\hline
\end{tabular}

Abbreviations: HDL-C, high-density lipoprotein cholesterol; LDL-C, low-density lipoprotein cholesterol; V/HCTZ, valsartan/hydrochlorothiazide; V-high, valsartan $160 \mathrm{mg}$; V-low, valsartan $80 \mathrm{mg}$.

${ }^{*} P<0.05$ vs baseline; ${ }^{\dagger} P<0.05$ vs V-low.

reductions $(P<0.05)$ for V/HCTZ $\left(-0.19 \mathrm{mmol}^{-1}\right)$ as compared with V-low $\left(-0.09 \mathrm{mmol}^{-1}\right)$

Serious AEs were experienced by five patients $(2.3 \%)$ in the V-low group, three patients $(1.4 \%)$ in the V-high group and five patients $(2.3 \%)$ in the V/HCTZ group. Seventeen $(2.6 \%)$ patients experienced AEs or SAEs, causing discontinuation from the study: $6(2.8 \%)$ in the V-low, $5(2.3 \%)$ in the V-high and $6(2.8 \%)$ in the V/HCTZ group. Suspected AEs leading to withdrawal from the study included dizziness, headache, anxiety, asthenia, fatigue and nausea, and were distributed among the three treatment groups. There were no drug discontinuations for hypotension in the study. There were no deaths reported in this study.

\section{Discussion}

Although evidence from clinical trials suggests that $\mathrm{BP}$ reduction to goal is crucial in reducing CV outcomes, at least half of all hypertensives remain untreated, with most of those that are treated requiring combination therapy to achieve the target BP control rate. ${ }^{1,6}$ Results from this study suggest that for patients with stage-1 and stage-2 hypertension, initiating treatment with combination therapy using the ARB, valsartan and low-dose HCTZ (160/ $12.5 \mathrm{mg}$ and increasing the dose to $160 / 25 \mathrm{mg}$ if needed) provided greater benefit in reaching $\mathrm{BP}$ control rates compared with initiating treatment with low- or high-dose valsartan monotherapy. The study demonstrated that by initiating therapy with two antihypertensive medications and titrating the dose every 2 weeks, if required, will lead to more effective BP control rates in a shorter period of time as compared with initiating treatment by monotherapy. After only 4 weeks of therapy, 58\% of patients on the initial combination agent achieved BP control as compared with only $26 \%$ initiated with valsartan (80 mg). Furthermore, AEs between V/HCTZ and the two valsartan monotherapy arms were similar, confirming the excellent tolerability achieved with 
combination of an ARB and low-dose diuretic. Risks associated with excessive drops in BP in V/HCTZ were also not observed, as noted by similar incidences of AEs related to low $\mathrm{BP}$ and hypotensive episodes among the three groups during the study. The preliminary concern of 'over-treatment' in the V/HCTZ group was also not observed, since over $85 \%$ of patients initiated on valsartan monotherapy required add-on HCTZ therapy.

In the Valsartan Antihypertensive Long-term Use Evaluation (VALUE) study, which compared the CV outcomes of 15314 eligible patients randomized to a valsartan or an amlodipine-based treatment, higher odds ratios in favour of amlodipine were noted for all endpoints during the first 6 months, when BP differences between the treatment groups were greatest. ${ }^{12}$ The starting dose of valsartan in the VALUE trial was $80 \mathrm{mg}$. Patients remained on the valsartan $80-\mathrm{mg}$ dose for at least 1 month (4 weeks) before being up-titrated to the next step in the protocol. In our current study, initiating treatment with higher dose valsartan monotherapy $(160 \mathrm{mg})$ was associated with higher BP control rates and a shortened time to reach BP control as compared with low-dose valsartan $(80 \mathrm{mg})$ monotherapy, but the BP differences were largely influenced by addition of low-dose HCTZ at week 4 (Figure 4). Perhaps the early outcomes of the VALUE trial were not favourable for valsartan as the trial not only underestimated the proportion of patients requiring combination therapy, but also underutilized the add-on of HCTZ ( $40 \%$ of patients in the trial stayed on valsartan monotherapy, with only $70 \%$ of those patients having their BP controlled).

Studies with initial combination therapy using an ARB and low-dose HCTZ have demonstrated superior BP control rates and BP-lowering efficacy as compared with initiating therapy with monotherapy in patients with stage-2 hypertension or diabetes. $^{4,5,13-16}$ There is a consensus that initial combination therapy works for high-risk patients with stage-2 hypertension; however, this does not have current support as an optional treatment approach for wider use in primary hypertension. ${ }^{11}$ Evidence supporting initial use of combination therapy for patients with stage-1 hypertension largely comes from recent findings from the ACCOMPLISH study. ${ }^{17}$ Over 9000 patients with stage-1 or stage-2 hypertension were initiated with ACE-inhibitor + HCTZ or ACE-inhibitor + amlodipine combination therapy. The study demonstrated that use of initial combination therapy was very effective and safe for both stage- 1 and stage-2 hypertension, as it reported some of the highest $\mathrm{BP}$ control rates for any large outcomes study. ${ }^{18}$

One of the clinical challenges with treating a multifactorial disease like hypertension is that it is very difficult to predict an individual's response to any given treatment. An individual's response to therapy is not only influenced by baseline factors, such as activity of the renin-angiotensin system and sodium intake, but is also affected by the patient's compensatory response to the therapy in an attempt to prevent further lowering BP. Using two welltolerated antihypertensive agents with complementary modes of action to reduce BP will act to limit the counter-regulatory response and thus ensure effective antihypertensive response in a majority of patients. ${ }^{19}$ Initiating antihypertensive therapy for patients with stage-1 and stage- 2 hypertension, using a well-tolerated combination agent such as an ACE-inhibitor or ARB and low-dose diuretic, is perhaps a more effective approach to enable more patients in the primary care setting to reach the BP goal. ${ }^{17} \mathrm{~A}$ recent study adopted this treatment approach in primary care offices where patients were initiated with combination therapy using a renin-angiotensin system blocker (ACE inhibitor or $\mathrm{ARB}$ ) and low-dose diuretic as compared with the conventional guideline treatment approach of starting with monotherapy. ${ }^{20}$ The study demonstrated excellent tolerability and superior BP control rates using initial combination therapy as compared with those patients who were started with monotherapy and titrated upwards following established treatment guidelines. Thus there is good evidence to recommend wide use of combination agents as firstline therapy for hypertensive patients, regardless of their baseline BP, in the clinical practice setting.

\section{Study limitations}

This study was not designed to demonstrate the complete treatment strategy approach for using a valsartan-based regimen, as it did not allow achievement of the maximum antihypertensive effect. The study did not include the maximum dose of valsartan $(320 \mathrm{mg})$ in combination with HCTZ, was of 6 weeks duration and the dose titration period was only 2 weeks. The study, rather, was designed to test the efficacy and tolerability of using initial combination therapy for patients with primary stage-1 and stage-2 hypertension.

In conclusion, initial use of combination therapy using a renin-angiotensin system blocker and lowdose diuretic in the standard treatment of hypertension in the primary care setting is an idea whose time has not yet come but which finds support in this study. In this study, initiating therapy with valsartan and low-dose HCTZ (160/12.5 mg) resulted in higher BP control rates, greater antihypertensive effect and prompt BP control with no significant increase in AEs in patients with stage- 1 or stage- 2 hypertension. The study has provided a template one could follow for treating hypertension effectively and safely using initial combination therapy, including uncomplicated cases of stage-1 to stage-2 hypertension. This study has demonstrated that initial combination therapy with an ARB and low-dose diuretic should become routine for treatment of hypertension, thereby improving BP control rates for patients with primary, essential hypertension. 
What is known about the topic

- Earlier and more frequent use of combination therapy in the management of hypertension would greatly improve BP control rates.

- The guidelines mention that patients with baseline BP measures more than $20 \mathrm{~mm} \mathrm{Hg}$ above the goal (that is, stage-2 hypertension) are candidates for initial combination therapy as they would not have their BP controlled by monotherapy.

- In patients unresponsive to valsartan or low-dose HCTZ monotherapy, addition of another agent (addition of HCTZ to valsartan or vice versa) has resulted in further reductions of $\mathrm{BP}$ without increase in AEs.

What this study adds

- This study examined the relative benefits of initiating combination therapy with valsartan+low-dose HCTZ as compared with valsartan monotherapy for patients with mild-to-moderate (stage-1 or 2) uncomplicated hypertension.

- This study has provided strong support for wide use of combination agents as first-line therapy for hypertensive patients, regardless of their baseline BP, in the clinical practice setting.

- Safety concerns about initiating combination therapy for patients with primary hypertension have been alleviated through use of an ARB combined with a low-dose thiazide diuretic.

Abbreviations: $\mathrm{AE}$, adverse event; $\mathrm{ARB}$, angiotensin-receptor blocker; $\mathrm{BP}$, blood pressure; HCTZ, hydrochlorothiazide.

\section{Conflict of interest}

KAJ: Consultant for Novartis Pharmaceuticals Corporation, Merck and Daiichi Sankyo; received lecture fees from Novartis Pharmaceuticals Corporation, Abbott, Bristol-Myers Squibb, GlaxoSmithKline and Merck; received research support from Novartis Pharmaceuticals Corporation and King Pharmaceuticals.

BFP: Consultant for Novartis Pharmaceuticals Inc.; received lecture fees from Astellas Pharmaceuticals, Boehringer Ingelheim Pharmaceuticals Inc. and Novartis Pharmaceuticals Corporation.

DC: Consultant for Novartis Pharmaceuticals Inc.; served as clinical trial investigator for Novartis Pharmaceuticals Corporation, Astra-Zeneca, Daiichi Sankyo and Pfizer.

DZ/DP/RS: Employed at Novartis.

\section{Acknowledgements}

This study was supported by Novartis Pharmaceuticals Corporation. We acknowledge the support of all participating investigators. The sponsor was responsible for the design and conduct of the study, and collection and management of data. The authors were responsible for the preparation, review and final approval of the paper. We thank Novartis employee, Kanaka Sridharan, MS, RPh, for editorial assistance. We express our appreciation to Ms LaTonya Collins, BN, of Novartis Pharmaceuticals Corporation for expert assistance in project management (Clinical Trials.gov identifier NCT00280540).

\section{References}

1 Ong KL, Cheung BM, Man YB, Lau CP, Lam KS. Prevalence, awareness, treatment, and control of hypertension among United States adults 1999-2004. Hypertension 2007; 49: 69-75.

2 Dahlöf B, Sever PS, Poulter NR, Wedel H, Beevers DG, Caulfield M, et al., for ASCOT Investigators. Prevention of cardiovascular events with an antihypertensive regimen of amlodipine adding perindopril as required versus atenolol adding bendroflumethiazide as required, in the Anglo-Scandinavian Cardiac Outcomes Trial-Blood Pressure Lowering Arm (ASCOT-BPLA): a multicentre randomized controlled trial. Lancet 2005; 366: 895-906.

3 Okonofua EC, Simpson KN, Jesri A, Rehman SU, Durkalski VL, Egan BM. Therapeutic inertia is an impediment to achieving the Healthy People 2010 blood pressure control goals. Hypertension 2006; 47: 345-351.

4 Neutel JM, Franklin SS, Oparil S, Bhaumik A, Ptaszynska A, Lapuerta P. Efficacy and safety of irbesartan/HCTZ combination therapy as initial treatment for rapid control of severe hypertension. J Clin Hypertens 2006; 8: 850-857.

5 Calhoun DA, Glazer RD, Pettyjohn FS, Coenen PD, Zhao Y, Grosso A. Efficacy and tolerability of combination therapy with valsartan/hydrochlorothiazide in the initial treatment of severe hypertension. Curr Med Res Opin 2008; 24: 2303-2311.

6 Chobanian AV, Bakris GL, Black HR, Cushman WC, Green LA, Izzo JL, et al., the National High Blood Pressure Education Program Coordinating Committee. Seventh report of the Joint National Committee on Prevention, Detection, Evaluation, and Treatment of High Blood Pressure. Hypertension 2003; 42: 1206-1252.

7 Mancia G, De Backer G, Dominiczak A, Cifkova R, Fagard R, Germano G et al. Management of Arterial Hypertension of the European Society of Hypertension; European Society of Cardiology. 2007 Guidelines for the Management of Arterial Hypertension: the Task Force for the Management of Arterial Hypertension of the European Society of Hypertension (ESH) and of the European Society of Cardiology (ESC). J Hypertens 2007; 25: 1105-1187.

8 Schmidt A, Adam SA, Kolloch R, Weidinger G, Handrock R. Antihypertensive effects of valsartan/ hydrochlorothiazide combination in essential hypertension. Blood Press 2001; 10: 230-237.

9 Lacourcière Y, Poirier L, Hebert D, Assouline L, Stolt P, Rehel B et al. Antihypertensive efficacy and tolerability of two fixed-dose combinations of valsartan and hydrochlorothiazide compared with valsartan monotherapy in patients with stage 2 or 3 systolic hypertension: an 8-week, randomized, double-blind, parallelgroup trial. Clin Ther 2005; 27: 1013-1021.

10 White WB, Calhoun DA, Samuel R, Taylor AA, Zappe DH, Purkayastha D. Improving blood pressure control: increase the dose of diuretic or switch to a fixed-dose angiotensin receptor blocker/diuretic? The valsartan hydrochlorothiazide diuretic for initial control and titration to achieve optimal therapeutic effect (Val-DICTATE) trial. J Clin Hypertens 2008; 10: 450-458.

11 White WB. Improving blood pressure control and clinical outcomes through initial use of combination 
therapy in stage 2 hypertension. Blood Press Monit 2008; 13: 123-129.

12 Julius S, Kjeldsen SE, Weber M, Brunner HR, Ekman S, Hansson L, et al., for VALUE trial group. Outcomes in hypertensive patients at high cardiovascular risk treated with regimens based on valsartan or amlodipine: the VALUE randomised trial. Lancet 2004; 363: 2022-2031.

13 Franklin SS, Neutel JM. Initial combination therapy for rapid and effective control of moderate and severe hypertension. J Hum Hypertens 2009; 23: 4-11.

14 Franklin S, Lapuerta P, Cox D, Donovan M. Initial combination therapy with irbesartan/hydrochlorothiazide for hypertension: an analysis of the relationship between baseline blood pressure and the need for combination therapy. J Clin Hypertens 2007; 9(Suppl 5): 15-22.

15 ADVANCE Collaborative GroupPatel A, MacMahon S, Chalmers J, Neal B, Woodward M, Billot L et al. Effects of a fixed combination of perindopril and indapamide on macrovascular and microvascular outcomes in patients with type 2 diabetes mellitus (the ADVANCE trial): a randomised controlled trial. Lancet 2007; 370: 829-840.

16 Lacourcière Y, Poirier L, Lefebvre J. Expedited blood pressure control with initial angiotensin II antagonist/ diuretic therapy compared with stepped-care therapy in patients with ambulatory systolic hypertension. Can J Cardiol 2007; 23: 377-382.

17 Welsh L, Ferro A. Drug treatment of essential hypertension: the case for initial combination therapy. Int $J$ Clin Pract 2004; 58: 956-963.

18 Jamerson K, Bakris GL, Dahlöf B, Pitt B, Velazquez E, Gupte J, et al., for ACCOMPLISH Investigators. Exceptional early blood pressure control rates: the ACCOMPLISH trial. Blood Press 2007; 16: 80-86.

19 Palmer BF. Improving BP control with combined renin-angiotensin system blockade and thiazide diuretics in hypertensive patients with diabetes mellitus or kidney disease. Am J Cardiovasc Drugs 2008; 8: 9-14.

20 Feldman RD, Zou GY, Vandervoort MK, Wong CJ, Nelson SA, Feagan BG. A simplified approach to the treatment of uncomplicated hypertension: a cluster randomized, controlled trial. Hypertension 2009; 53: 646-653.

This work is licensed under the Creative Commons Attribution-NonCommercialNo Derivative Works 3.0 License. To view a copy of this license, visit http://creativecommons.org/licenses/ by-nc-nd/3.0/ 